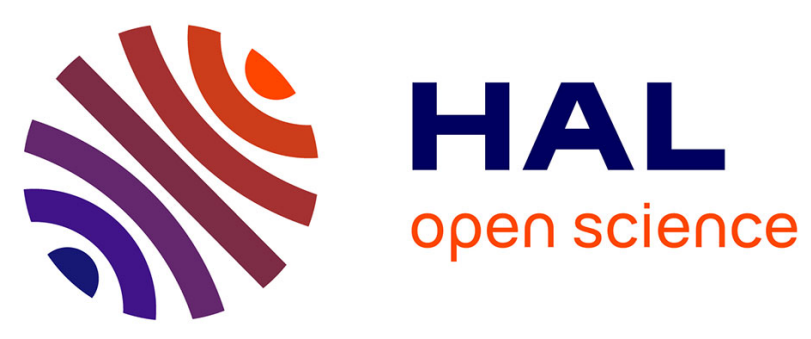

\title{
What is Partial Ambiguity?
}

Loïc Berger

\section{To cite this version:}

Loïc Berger. What is Partial Ambiguity?. Economics and Philosophy, 2022, 38 (2), pp.206-220. hal-03158580

\section{HAL Id: hal-03158580 \\ https://hal.science/hal-03158580}

Submitted on 4 Mar 2021

HAL is a multi-disciplinary open access archive for the deposit and dissemination of scientific research documents, whether they are published or not. The documents may come from teaching and research institutions in France or abroad, or from public or private research centers.
L'archive ouverte pluridisciplinaire HAL, est destinée au dépôt et à la diffusion de documents scientifiques de niveau recherche, publiés ou non, émanant des établissements d'enseignement et de recherche français ou étrangers, des laboratoires publics ou privés. 


\title{
WHAT IS PARTIAL AMBIGUITY?
}

\author{
Loïc Berger*
}

March 4, 2021

\begin{abstract}
This paper reflects on the notion of partial ambiguity. Using a framework decomposing ambiguity into distinct layers of analysis, among which are risk and model uncertainty, and allowing for different attitudes toward these layers, I show that partial ambiguity may prove less desirable than full ambiguity, even under ambiguity aversion. This observation poses difficulties for interpreting the notion of partial ambiguity in relation to the partial information available to determine the potential compositions of an ambiguous urn. Two Ellsberg-style thought experiments are described to challenge the meaning of partial ambiguity further, and an alternative interpretation, based on a more ambiguous relation, is discussed.
\end{abstract}

Keywords: Ambiguity, model uncertainty, smooth ambiguity aversion, Ellsberg paradox

\section{JEL Classification: D 81}

\footnotetext{
${ }^{*}$ CNRS, IESEG School of Management, Univ. Lille, UMR 9221-LEM, F-59000 Lille, France; and Bocconi University, Italy - E-mail address: l.berger@ieseg.fr. This research is supported by a grant from the French Agence Nationale de la Recherche (ANR-17-CE03-0008-01 INDUCED) and from the European Research Council (ERC-2013-StG 336703-RISICO). I am grateful to Mohammed Abdellaoui, Ilke Aydogan, Aurélien Baillon, David Crainich, Simone Cerreia-Vioglio, Louis Eeckhoudt, Itzhak Gilboa, Sujoy Mukerji, Peter Wakker and especially Massimo Marinacci for helpful comments and discussions.
} 
Ambiguity may be high even where there is ample quantity of information [...]

(Ellsberg, 1961, p. 659)

\section{Introduction}

Ambiguity is the term that has emerged in the literature since the seminal paper of Ellsberg (1961) to characterize situations in which the probabilities of uncertain events are unknown. Ambiguity is present in virtually all real-life situations and plays a significant role in most economic problems. It is distinct from the notion of risk, which refers to situations in which probabilities are perfectly known.

In a recent study, Chew, Miao, and Zhong (2017) extended the decision-making problem originally presented in Ellsberg (1961) by proposing different forms of "partial ambiguity". These authors write (p. 1240):

"As espoused by Knight, the essence of decision making under uncertainty is partial knowledge that may arise from vague evidence, diverse information, or conflicting news. Going beyond pure risk and full ambiguity in the original two-urn paradox, this paper experimentally studies decision making in a richer domain of uncertainty involving intermediate forms of ambiguity."

The interpretation giving rise to such a notion of partial ambiguity implicitly relies on the partial information, or knowledge, available to pin down the potential probability models describing the stochastic phenomenon of interest: from 1, in the pure risk case, to 101, in the full ambiguity case, originated from Ellsberg (1961).

In this paper, I reflect on the meaning of partial ambiguity as an intermediate form of ambiguity. Using a recently developed approach that decomposes ambiguity into distinct layers of analysis, among which are risk and model uncertainty (Hansen, 2014; Marinacci, 2015; Hansen and Marinacci, 2016), and that allows for distinct layers to be treated differently, I show that partial ambiguity may be deemed less desirable than full ambiguity to an ambiguity-averse decision maker (DM). ${ }^{1}$ This counterintuitive observation challenges the significance of partial ambiguity.

In line with the risk theory literature (for which the notion of partial risk does not exist), I argue that partial ambiguity may not be helpful in characterizing more precisely uncertain situations that are by nature either risky or ambiguous, but hardly admit intermediate forms between them (i.e., either the probability distribution is known or it is unknown). However, if a refinement needs to be made in the classification of different ambiguous situations, I propose that a natural candidate can characterize an ambiguous act as whether it is affected more by ambiguity than another. This classification could be based on the more ambiguous notion proposed by Jewitt and Mukerji (2017). It has the

\footnotetext{
${ }^{1}$ Note that, technically, this result coincides with Predictions 1 and 2 in Chew, Miao, and Zhong (2017).
} 
advantage of mirroring the well-established notion of more risky (Rothschild and Stiglitz, 1970) widely used in the risk theory literature. It furthermore allows for establishing a partial ordering among ambiguous situations (within a given class of preferences).

\section{Ambiguity and the layers of uncertainty}

\subsection{Ellsberg paradox}

Ellsberg (1961) proposed two thought-experiment decision problems, which have been the primary examples motivating research on ambiguity. One of them, known as the two-color problem, goes as follows. ${ }^{2}$

Thought Experiment [Ellsberg, 1961] Imagine that you confront two urns containing red and black balls, from one of which a ball will be drawn at random. You can choose the color and the urn on which to bet. With a correct bet, you win $\$ 100$ (and nothing happens if your bet is incorrect). You have the following information. The first urn, called E100, contains 100 red and black balls, but in a ratio entirely unknown to you. The second urn, called R100, contains exactly 50 red and 50 black balls. On which urn do you prefer to bet?

The four possible event-contingent payment schemes of the problem are presented in Table 1. They depend on the color of the ball drawn from one of the urns. Typically,

\begin{tabular}{lccccc}
\hline \hline & \multicolumn{2}{c}{$E 100$} & & \multicolumn{2}{c}{$R 100$} \\
\cline { 2 - 3 } \cline { 5 - 6 } & Red & Black & & Red & Black \\
\hline$f$ & $\$ 100$ & $\$ 0$ & & $\$ 100$ & $\$ 0$ \\
$g$ & $\$ 0$ & $\$ 100$ & & & $\$ 100$ \\
$f^{\prime}$ & & & & $\$ 0$ & $\$$ \\
$g^{\prime}$ & & & & & \\
\hline
\end{tabular}

Table 1: Ellsberg two-color problem

what is observed is that people are indifferent on the color on which to bet: they express indifference between $f$ and $f^{\prime}$, and between $g$ and $g^{\prime}$ (which is noted $f \sim f^{\prime}$ and $g \sim g^{\prime}$, respectively); but prefer betting on $R 100$ rather than on $E 100$ : they express a preference for $g$ over $f$ and for $g^{\prime}$ over $f^{\prime}$ (noted $g \succ f$ and $g^{\prime} \succ f^{\prime}$ ). Such preferences are, however, incompatible with the existence of subjective probabilities: the ranking $f \prec g$ would imply that the probability of drawing a red ball in urn E100 is considered to be less than 0.5 (i.e., less than the probability of drawing a red ball in urn $R 100$ ), while at the same time the ranking $f^{\prime} \prec g^{\prime}$ would imply that the probability of drawing a black ball in urn E100

\footnotetext{
${ }^{2}$ Note that this thought experiment had already appeared in Keynes (1921).
} 
is considered to be less than 0.5 (i.e., less than the probability of drawing a black ball in $R 100)$. Overall, this would result in having the probabilities of complementary events summing up to less than 1 , which is inconsistent. As bets $f$ and $f^{\prime}$ involve ambiguity, whereas bets $g$ and $g^{\prime}$ involve risk, these typically observed preferences indicate ambiguity aversion, that is, a preference for known-probability bets over unknown ones.

\subsection{Decomposing ambiguity into layers of uncertainty}

Following the works of Hansen (2014); Marinacci (2015); and Hansen and Marinacci (2016), themselves building on early insights of Arrow (1951), ambiguity can generally be decomposed into different layers of uncertainty: (i) risk within a model, in which the uncertainty is about the outcomes of a given (probability) model that specifies fully the outcome probabilities; (ii) model uncertainty, in which the uncertainty is about which probability model (among a given set) should be used to assign these probabilities; and (iii) model misspecification, in which the uncertainty is induced by whether or not the correct model lies among the set under consideration (Aydogan et al., 2020). While most uncertain situations in real-life usually encompass the three layers of uncertainty together, Ellsberg's (1961) examples are particularly remarkable because they generate ambiguity using only the layers of type (i) and (ii) (indeed, model misspecification is nonexistent in Ellsberg's setup). For example, in the two-color problem described above, the composition of the urn E100 is necessarily one of the 101 compositions physically possible. ${ }^{3}$

The main distinction between the layers of risk and model uncertainty is the nature of the uncertainty they feature. A risk represents an objectively uncertain prospect. It refers to the physical quantification of uncertainty by means of a probability model (and as such is sometimes referred to as an instance of physical or aleatory uncertainty). It is usually referred to as a lottery, represented by the gamble $L=\left(x_{1}, p_{1} ; \ldots ; x_{n}, p_{n}\right)$ that yields outcome $x_{i}$ with a well-defined objective probability $p_{i}$. This probability $p_{i}$ is a measure of randomness. For example, a risk may be characterized by a specific composition of an urn from which a ball is randomly drawn. If the balls in the urn can only have two colors (e.g., red and black), the risk may further be characterized by the binary lottery $x_{p} y$, yielding $x$ with probability $p$ and $y$ otherwise.

The uncertainty about which probability model (or risk) should be considered is not of the same nature. ${ }^{4}$ In Ellsberg's problem, this second layer of uncertainty has an epistemic nature, as no objective probabilities may be assigned to the possible compositions of the urn. The prospect determining the correct composition of the urn is thus subjectively uncertain. In theory, a DM can still form a probability measure over the possible urn compositions, but these probabilities are necessarily subjective, reflecting the DM's degree of

\footnotetext{
${ }^{3}$ The number of red (black) balls in the urn necessarily belongs to $\{0,1,2, \ldots, 100\}$.

${ }^{4}$ Except in the case of compound risk, in which there is an objective probability distribution on the possible compositions of the urn. In this case, the compound lottery takes the form $\left(\ldots ; L_{i}, p_{i} ; \ldots\right)$, yielding the lottery $L_{i}=\left(\ldots ; x_{i j}, p_{i j} ; \ldots\right)$ with probability $p_{i}$.
} 
belief in each possible model. Under this decomposition of ambiguity into different layers, ambiguity aversion results from a preference for risk over model uncertainty (see Marinacci, 2015; for a theoretical discussion, and Berger and Bosetti, 2020; for experimental evidence).

\section{$3 \quad$ Ambiguity theories}

In this section, I shortly outline different approaches economists have developed to deal with ambiguity under the two-layer decomposition. I focus on two approaches that adopt a Bayesian perspective in that they quantify the uncertainty within each layer using a single probability measure.

Consider a finite set $S$ of states of the environment and a set $C$ of consequences. (Technical details are standard and are omitted here.) Adopting Savage's (1954) approach, an act is defined as a choice with state-contingent consequences, that is a mapping a : $S \rightarrow$ $C$. The collection of all acts is denoted by A. It is assumed that the DM has a preference relation $\succsim$ over acts, a binary relation that describes how she ranks alternatives. In particular, I write $\mathrm{a} \succsim \mathrm{b}$ if the DM either strictly prefers act a to act $\mathrm{b}$ or is indifferent between the two. As usual, $\sim$ denotes indifference and $\succ$ strict preference. I assmue that the DM is able, because of her ex ante information, to posit a set of potential models $M$ describing the likelihoods of the different states. This set of models is taken as a datum of the decision problem: the DM behaves as if she knows that states are generated by a probability model $m$ that belongs to the collection $M$. Each probability model $m$ therefore describes a given risk and, as such, represents the intrinsic randomness that states feature. The epistemic uncertainty is about which is the correct model among the collection $M$. I write $M=\left\{m_{\theta}\right\}$, indicating that each model is indexed by a parameter $\theta$. For example, the Ellsberg's urn $E 100$ has 101 possible compositions, which can be summarized by $M=\left\{m_{\theta}=\frac{\theta}{100}\right.$ for $\left.\theta \in\{0,1, \ldots, 100\}\right\} .5$ The (Savage) decision problem under uncertainty is fully characterized by the sets of acts, states, consequences, and models, together with the preference relation, that is, the quintet $(\mathrm{A}, S, C, M, \succsim)$.

\subsection{Subjective expected utility}

Under the benchmark approach of subjective expected utility (SEU), a preference satisfying Savage's (1954) axioms is represented by the criterion

$$
U_{S E U}(\mathrm{a})=\sum_{\theta}\left(\sum_{s} u(\mathrm{a}(s)) m_{\theta}(s)\right) \mu(\theta)=\sum_{s} u(\mathrm{a}(s)) \bar{m}(s) .
$$

In these expressions, $u$ is a von Neumann-Morgernstern utility function. It translates economic consequences, measured in monetary terms, into utility levels, and is known to

\footnotetext{
${ }^{5}$ In an abuse of notations, I let the model $m_{p}=x_{p} y$ be fully characterized by the probability $p$.
} 
capture risk attitudes. As shown within the bigger parentheses, it is possible, for each model $m_{\theta}$, to compute the expected payoff of a given act. This expected payoff takes an expected utility form: it is computed as the weighted sum of utilities obtained from the consequences in each state of the environment. To address model uncertainty, the DM has a subjective prior probability distribution $\mu$ that quantifies her belief about the true parameter $\theta$. Thus, $\mu(\theta)$ is the DM's subjective belief that $m_{\theta}$ is the true model. As the DM does not know which is the correct model, she considers the expected payoff of each possible model and aggregates them out by performing a weighted average according to the relative weights that she associates with each of them (i.e., her prior beliefs $\mu(\theta)$ ). The first representation hereabove is the two-layer version of SEU that has been axiomatized by Cerreia-Vioglio et al. (2013). Under this framework, the layers of risk and model uncertainty are implicitly treated in the same way. The second representation is the original reduced form of Savage (1954), in which $\bar{m}$ is the predictive probability induced by the prior $\mu: \bar{m}(s)=\sum_{\theta} m_{\theta}(s) \mu(\theta)$ for all states $s$. In Ellsberg's experiment, for example, a bet on the ambiguous urn $E 100$, giving $x=100$ if correct and $y=0$ otherwise, yields $U_{S E U}=\frac{1}{101} \sum_{\theta=0}^{100} \frac{\theta}{100} u(100)=\frac{1}{2} u(100)$, which is exactly the same as a bet on the risky urn $R 100 .^{6}$

\subsection{Smooth ambiguity}

To accommodate the phenomenon of ambiguity aversion highlighted in Ellsberg (1961), the theoretical economic literature has followed different perspectives. One of them, known as the smooth ambiguity approach (Klibanoff et al., 2005, KMM hereinafter), also adopts a Bayesian perspective by having the two layers of uncertainty quantified by a single probability measure. However, in contrast with SEU, a distinct treatment is allowed for the layers of risk and model uncertainty. By letting the function $v$ represent the DM's attitude toward model uncertainty, a natural generalization of the SEU criterion emerges as a version of the smooth ambiguity model of KMM. Under this framework, acts are ranked according to the criterion

$$
U_{K M M}(\mathrm{a})=\sum_{\theta} \phi\left(\sum_{s} u(\mathrm{a}(s)) m_{\theta}(s)\right) \mu(\theta) .
$$

A stronger aversion to the layer of model uncertainty than to that of risk leads to ambiguity aversion, modeled by a concave function $\phi=v \circ u^{-1}$. Under this criterion, a bet on urn $E 100$ yields $U_{K M M}=\frac{1}{101} \sum_{\theta=0}^{100} \phi\left(\frac{\theta}{100} u(100)\right)$, and it is easy to show that $U_{K M M} \leq \phi\left(U_{S E U}\right)$ under ambiguity aversion. This interpretation of a non-neutral attitude toward ambiguity stems from non-reduction of objectively and subjectively determined probabilistic judgements (i.e., different confidence in such judgments).

\footnotetext{
${ }^{6}$ Under the assumption of uniform prior $\mu$ and after normalizing $u(0)=0$.
} 


\subsection{Other approaches}

The smooth ambiguity approach allows for viewing ambiguity à la Ellsberg as a twostage uncertain prospect, in which the uncertainty in each stage corresponds to a distinct layer. Other approaches also model ambiguity as multiple stages of uncertainty while not necessarily making a distinction between the layers of risk and model uncertainty (see Aydogan et al., 2020, for more details). This is for example the case of the approaches proposed by Segal (1987) (recursive rank dependent utility approach, RRDU) and by Seo (2009). As these approaches do not distinguish between objectively and subjectively determined probabilistic judgements in the distinct layers, they made no distinction between compound risk (in which there are two stages of risk) and ambiguity à la Ellsberg (in which there are a layer of risk and a layer of model uncertainty). Hence, as non-neutral ambiguity attitudes may result from the violation of reduction of compound lotteries, an elementary rationality condition, these theories assign to ambiguity attitudes a purely descriptive status.

\section{Partial ambiguity}

Chew, Miao, and Zhong (2017) recently proposed the notion of partial ambiguity to describe the situations "going beyond pure risk and full ambiguity in the original two-urn paradox" (p. 1240). The "intermediate forms of ambiguity" they propose may be described using an urn containing 100 red or black balls. Specifically, by letting $n \in\{0,1, \ldots, 50\}$, partial ambiguity may take the form of:

- Interval Ambiguity, when the number of red (or black) balls is in $I_{n}=[50-n ; 50+n]$;

- Disjoint Ambiguity, when the number of red (or black) balls is in $D_{n}=[0 ; n] \cup[100-$ $n ; 100] ;$ and

- Two-point Ambiguity, when the number of red (or black) balls is in $T_{n}=\{50-n, 50+n\}$.

By letting $n$ vary between 0 and 50, these situations span the space of possible urn compositions between the risky urn $R 100$ (e.g., in the case $I_{0}=T_{0}$ ) in which there is no ambiguity, and Ellsberg's ambiguous urn E100 (e.g., in the case $I_{50}=D_{0}$ ), which is coined "full ambiguity" (Chew, Miao, and Zhong, 2017, p. 1240). Table 2 presents the sets $M$ of possible models (or urn compositions) induced by these three forms of partial ambiguity, for the cases $n=0, n=25$, and $n=50$.

To understand why these situations may be seen as involving partial forms of ambiguity, different interpretations may be considered. 
Table 2: Sets of Models in the Partial ambiguity Situations

\begin{tabular}{|c|c|c|c|}
\hline \multirow{2}{*}{$\begin{array}{l}\text { Types of } \\
\text { partial ambiguity }\end{array}$} & \multicolumn{3}{|c|}{$n$} \\
\hline & 0 & 25 & 50 \\
\hline$I_{n}$ & $\begin{array}{c}\left\{\frac{50}{100}\right\} \\
(1)\end{array}$ & $\begin{array}{c}\left\{\frac{25}{100}, \frac{26}{100}, \ldots, \frac{75}{100}\right\} \\
(51)\end{array}$ & $\begin{array}{c}\left\{\frac{0}{100}, \frac{1}{100}, \ldots, \frac{100}{100}\right\} \\
(101)\end{array}$ \\
\hline$D_{n}$ & $\begin{array}{l}\left\{\frac{0}{100}, \frac{100}{100}\right\} \\
(2)\end{array}$ & $\begin{array}{c}\left\{\frac{0}{100}, \ldots, \frac{25}{100}\right\} \cup\left\{\frac{75}{100}, \ldots, \frac{100}{100}\right\} \\
(52)\end{array}$ & $\begin{array}{c}\left\{\frac{0}{100}, \frac{1}{100}, \ldots, \frac{100}{100}\right\} \\
(101)\end{array}$ \\
\hline$T_{n}$ & $\left\{\frac{50}{100}\right\}$ & $\left\{\frac{25}{100}, \frac{75}{100}\right\}$ & $\begin{array}{l}\left\{\frac{0}{100}, \frac{100}{100}\right\} \\
(2)\end{array}$ \\
\hline
\end{tabular}

Note: Number of possible models in parenthesis $(|M|)$

\subsection{Numbers of potential models}

The first, natural, way to interpret these situations derives from the number of possible compositions an ambiguous urn may entail. From Table 2, we can observe that: ${ }^{7}$

Observation 1 The number of potential models, noted $|M|$, physically compatible with each form of partial ambiguity is increasing in $n$ (strictly increasing in the cases of $I_{n}$ and $D_{n}$, and weakly increasing -first increasing, then constant-in the case of $T_{n}$ ).

More specifically, the number of possible models lies between 1, when there is no ambiguity and 101, when there is full ambiguity. Under this interpretation, what makes a situation of partial ambiguity thus corresponds to lying between these boundaries: $|M| \in(1,101)$.

\subsection{Information and its structure}

Amount of information The partial ambiguity situations can also be analyzed in terms of the amount of information they carry. For example, in the case of interval ambiguity, the situations lie between one in which you know the color of each individual ball in the urn (in the no ambiguity situation, $I_{0}$ ) and a situation in which you do not know the color of any of the balls (in the full ambiguity situation, $I_{50}$ ). More generally, the information available in the different forms of partial ambiguity can be summarized as follows:

- In situations $I_{n}$, it is known that the urn is composed of at least $(50-n)$ red balls, and at least $(50-n)$ black balls,

- In situations $D_{n}$, it is known that at least $(100-n)$ balls in the urn have the same color,

\footnotetext{
${ }^{7}$ Remark that an analogous observation is present in Chew, Miao, and Zhong (2017) p. 1241.
} 
- In situations $T_{n}$, it is known that there are exactly either $(50-n)$ or $(50+n)$ red (or black) balls in the urn.

We can thus observe the following.

Observation 2 The amount of information available, within each form of partial ambiguity, is decreasing in $n$ (strictly decreasing in the cases of $I_{n}$ and $D_{n}$, and weakly decreasing-first decreasing, then constant-in the case of $T_{n}$ ).

Note that this interpretation, in terms of the amount of information, has sometimes been related to the one in terms of the numbers of potential models presented before. This is, for example, the case in the work of Einhorn and Hogarth (1985, p. 435), who wrote "Thus, ambiguity results from the uncertainty associated with specifying which of a set of distributions is appropriate in a given situation. Moreover, the amount of ambiguity is an increasing function of the number of distributions that are not ruled out (or made implausible) by one's knowledge of the situation."

Quality of information Alternatively, the partial ambiguity situations may also be presented in terms of the quality of information they carry, using their information structure. The DM's information about the likelihoods of the different states (and thus the outcome of the bet) is a priori modeled by a set $M$. By letting $S=\{r, b\}$ be the state space, in which the ball drawn from the urn is either red $(r)$ or black $(b)$, the set of models in each uncertain situation can be expressed as $M=\{m$ such that $P(r) \in I\}$. In this expression, $P(r)$ is the probability that the ball drawn is red, and $I \subseteq[0,1]$ is a set-theoretic modeling of information. It represents the available information characterizing the chances to make a correct bet. In urn $E 100$ for example, $I=\left\{\frac{0}{100}, \frac{1}{100}, \ldots, \frac{100}{100}\right\}$. Assuming that the DM has information about $M$, her acts needs to be measurable with respect to $M$. The set of models can then be partially ordered by the "finer than" and the "coarser than" relations:

Definition. If $M^{\prime}$ and $M$ are such that $I^{\prime}$ is a subset of $I$, then $M^{\prime}$ is said to be finer than $M$, and $M$ is said to be coarser than $M^{\prime}$.

In words, this means that better information regarding the structure of ambiguity may be naturally modeled by a smaller set of potential models. As such, a situation is called full ambiguity if its associated set of models is maximal as in E100, and is called partial ambiguity if its associated set of models is finer than in E100. We can then observe the following.

\section{Observation 3}

- In situations $I_{n}$ and $D_{n}$, the set of models becomes coarser as $n$ increases,

- The use of set theory remains silent on the ranking of situations under $T_{n}$. 
Regarding the second point, remark that the use of set theory does not enable us to distinguish $T_{25}$ from $T_{50}$, whose associated sets of models $M$ can be ranked by neither the finer nor the coarser relation.

\section{Challenges to partial ambiguity}

Rather than characterizing the notion of partial ambiguity in relation to the partial information available and the associated number of possible models, an alternative approach is to focus on how these situations are considered by individuals. Using the smooth ambiguity model discussed in Section 3, and following a simple stochastic dominance argument à la Rothschild and Stiglitz (1970), which has been widely used in economics, we can observe that, under a standard symmetry condition on the prior $\mu$, we have

\section{Proposition 1.}

- In situations $I_{n}$ and $T_{n}$, a smooth ambiguity-averse DM always strictly prefers a situation with a lower $n$,

- In situations $D_{n}$, a smooth ambiguity-averse DM always strictly prefers a situation with a higher $n$.

The intuition behind these results is simple. Under the smooth ambiguity model, ambiguity aversion can be interpreted as an aversion to mean preserving spreads (MPS) in the space of expected utilities induced by different urn compositions. It is then easy to see that, under a specific, but widely accepted, symmetry condition on the subjective probabilities associated with each model, ${ }^{8}$ a MPS pattern emerges as $n$ increases for $I_{n}$ and $T_{n}$, and as $n$ decreases for $D_{n}$. In line with a hedging motive against extreme compositions, such MPSs of the possible expected utilities are disliked by ambiguity-averse DMs. Remark also that, in the case of $I_{n}$, Proposition 1 encompasses the standard result of Ellsberg (1961) that the risky urn $R 100$ is preferred to the ambiguous urn E100 under ambiguity aversion, while in the case of $D_{n}$, a thought-provoking result arises from comparing partial ambiguity $\left(D_{n}\right.$ with $\left.n<50\right)$ with full ambiguity $\left(D_{50}\right)$.

Corollary 1. In situations $D_{n}$, a smooth ambiguity-averse DM always strictly prefers full ambiguity to partial ambiguity.

\footnotetext{
${ }^{8}$ As noted by Chew et al. (2017), uniform, binomial, geometric, and U-shaped priors are all sufficient, because they (1) are symmetric and (2) imply simple spreads as $n$ increases (in the case of $I_{n}$ and $T_{n}$ ) or decreases (in the case of $D_{n}$ ). These two properties together are sufficient to guarantee the MPS condition. Remark that these conditions on the prior are less demanding than they appear at first glance. The symmetry in the prior distribution stems from the indifference between betting on a red or black ball. In the same vein, a uniform distribution ensures the same treatment for all compositions of the urn that are physically possible, and a binomial distribution results from associating the same subjective belief to each individual ball being red or black. All these arguments may be justified on the grounds of a general symmetry of information argument: given the information available, there is a priori no reason to believe that one event deserves more weight than another. The prior measure therefore reflects this symmetry, in accordance with the Principle of Insufficient Reason.
} 
Considering Proposition 1 together with Observations 2 and 3 may thus be seen as challenging the significance of the notion of partial ambiguity. In particular, it implies that more information may either increase (in situations $I_{n}$ ) or decrease (in $D_{n}$ ) utility under ambiguity aversion. Similarly, based on the quality of the partial information available, a finer set of models does not necessarily increase the utility of a bet. As a consequence, an ambiguity-averse DM may be better off (1) when the information provided makes the set of models finer (e.g., in the case of $I_{n}$ ), (2) when it makes the set of models coarser (e.g., in the case of $D_{n}$ ), or (3) when it does not make the set of models finer nor coarser (e.g., in the case of $T_{n}$ ). Said differently, the value of information regarding the composition of the urn is not necessarily positive. ${ }^{9}$

This result may not be intuitive at first glance. In the case of $I_{n}$, it seems relatively straightforward that, starting from Ellsberg urn E100, in which nothing is known, and being told that, out of the 100 balls present in the urn, at least $(50-n)$ of them are red and at least $(50-n)$ are black (e.g. going from $I_{50}$ to $\left.I_{25}\right)$, increases the utility of the bet. This information indeed enables the DM to disregard more extreme compositions of the urn. However, in the case of $D_{n}$, starting from Ellsberg urn E100 and being told that at least $(100-n)$ balls in the urn have the same color (e.g., going from $D_{50}$ to $D_{n}$ ) decreases the utility of the bet under smooth ambiguity aversion. Having more information (and thus being able to restrict the set of potential models) in this case is therefore not valuable.

Ultimately, what matters for decision-making is the quality, rather than the quantity, of information. As noted by Ellsberg (1961, p. 659), "ambiguity may be high even where there is ample quantity of information, where there are questions of reliability and relevance of information, and particularly where there is conflicting opinion and evidence."

In what follows, we propose two thought experiments that further challenge the notion of partial ambiguity. First, consider the situation $D_{0}=T_{50}$, which is characterized by the set of models $M=\left\{\frac{0}{100}, \frac{100}{100}\right\}$. As discussed earlier, it might be argued that, in this situation, partial information is available (i.e., the 100 balls have all the same color) compared with the urn E100, for which there is no information. However, does that necessarily imply that such a situation is of partial ambiguity as opposed to the full ambiguity situation E100? Here is an alternative way to present the problem.

\footnotetext{
${ }^{9}$ The value of information may for example be defined as the amount of money that a DM would be willing to pay for information about the composition of the urn (i.e. for a finer set $M^{\prime}$ ) before making a decision. While in general, information is seen as a way to reduce uncertainty -which has thus a positive economic value- it is worth noting that, in some contexts, information may also increase uncertainty, so that individuals may be inclined to avoid it. This is the case in the example provided above, as well as in the context of updating under ambiguity. Indeed, a key concept known as "dilation" of sets of priors, familiar to common models of updating under ambiguity, is that information may increase relevant ambiguity, and thus make individuals worse off (Shishkin and Ortoleva, 2020). In that case, the set of relevant priors may indeed become larger (dilate) after information. In a recent experimental study in this context, Kops and Pasichnichenko (2020) show that information aversion was significantly correlated with ambiguity aversion.
} 
Thought Experiment 1 Imagine that you confront two urns containing red and black balls, from one of which a ball will be drawn at random. You can choose the color and the urn on which to bet. With a correct bet, you win $\$ 100$ (and nothing happens if your bet is incorrect). You have the following information. The first urn, called E100, contains 100 red and black balls, but in a ratio entirely unknown to you. The second urn, called E1, contains only 1 ball that may be either red or black. On which urn do you prefer to bet?

If the answer to this question is $E 1$, then facing the bet with two models $M=\left\{\frac{0}{100}, \frac{100}{100}\right\}$ is deemed more desirable than facing the bet with 101 models $M=\left\{\frac{0}{100}, \frac{1}{100}, \ldots, \frac{100}{100}\right\}$. However, there is no reason, in this case, to consider that some (partial) information is available in $E 1$ compared with $E 100$ (the only difference between the two urns being their total number of balls). Instead, if, following the simple stochastic dominance argument exposed above, the answer is E100, then we need to conclude that, in the terminology proposed by Chew et al. (2017), a preference is expressed for full ambiguity over partial ambiguity. This counterintuitive result challenges the actual meaning of partial ambiguity. Second, it is also unclear what is the exact meaning of full ambiguity. To understand why, consider the following experiment.

Thought Experiment 2 Imagine that you confront two urns containing red and black balls, from one of which a ball will be drawn at random. You can choose the color and the urn on which to bet. With a correct bet, you win $\$ 100$ (and nothing happens if your bet is incorrect). You have the following information. The first urn, E100, contains 100 red and black balls, but in a ratio entirely unknown to you. The second urn, E1000, contains 1000 red and black balls, but also in a ratio entirely unknown to you. On which urn do you prefer to bet?

In this experiment, which of the two urns represents the situation of partial ambiguity, and which urn represents full ambiguity? Following the terminology adopted in Chew et al. (2017), E100 is the full ambiguity situation. However, following the line of arguments exposed above, E100-which entails 101 models-may be seen as encompassing partial information compared with E1000, which entails 1001 possible models. Indeed, remark that $E 100$ could be taken as a special case of $E 1000$, in which the DM is told that the 1000 balls are gathered in groups of 10 balls of the same color. Such type of partial information enables the DM to restrict the set of potential models to take in consideration (in terms of the sets modeling the information, we indeed have that $I_{E 100}$ is a subset of $I_{E 1000}$ ). Does it then imply that E100 is now the partial ambiguity situation and E1000 the full ambiguity situation? Pushing the reasoning further, how should be called the situation of an urn containing even more balls (e.g., 10,000)? In the limiting case, wouldn't the hypothetical ambiguous urn with an infinite number of balls (or Schmeidler's (1989) coin ${ }^{10}$ ) be

\footnotetext{
${ }^{10}$ Schmeidler (1989) proposed an example in which an individual is given the choice between betting on
} 
the only true instance of full ambiguity, as it is the only instance in which $I=[0,1]$ ? All ambiguous urns containing finite numbers of balls could indeed be viewed as instances of partial forms of ambiguity lying between such a full ambiguity urn and a risky urn.

\section{Discussion}

The two thought experiments presented in the previous section illustrate why a classification of intermediate forms of ambiguity based on the notion of partial ambiguity may be difficult to adopt. First, as an implicit parallelism is made between the notion of partial ambiguity and the partial information available, it might be believed that partial ambiguity conveys a notion of desirability it does not have with respect to the full ambiguity situation. As argued previously, and contrary to what economic intuition would suggest, there may well be situations in which providing more information actually decreases the desirability of the bet, so that full ambiguity is preferred to partial ambiguity under ambiguity aversion. Second, as Thought Experiments 1 and 2 indicate, it might not be consistent to call partial ambiguity a given situation in a specific context in which it is compared with other ambiguous situations, while the same situation (i.e., a situation with the same mathematical properties, which is associated with the same set $M$ of possible models) framed differently may have little, or nothing, to do with partial ambiguity. More fundamentally, partial ambiguity is a relative notion that is defined in opposition to full ambiguity, which has itself no clear meaning.

Overall, it is therefore unclear how the notion of partial ambiguity may be helpful in characterizing further uncertain situations, which typically are either risky (if the probability distribution is objectively known) or ambiguous (if the probability distribution is unknown), but hardly admit "partial" forms between them. However, if one thinks that an extra refinement needs to be made in the classification of different ambiguous situations, a natural candidate could consist in characterizing an ambiguous act as whether it is more affected by ambiguity than another. As Ellsberg (1961, p. 660-661) noted, "ambiguity is a subjective variable, but it should be possible to identify "objectively" some situations likely to present high ambiguity, by noting situations where available information is scanty or obviously unreliable or highly conflicting; or where expressed expectations of different individuals differ widely; or where expressed confidence in estimates tends to be low."

Such a classification of ambiguous situations could for example be based on the notion of more ambiguous proposed Jewitt and Mukerji (2017). It allows for establishing a partial ordering among ambiguous situations (within a given class of preferences), and has the advantage of mirroring the well established notion of more risky (Rothschild and Stiglitz, 1970), which has been widely used in the risk theory literature (note that in that literature,

the result of a known fair coin coming up Heads or Tails and betting on a coin that has never been tested and is absolutely unknown. Observe that in the latter case, the set of possible models is both continuous and bounded between 0 and 1 . 
the notion of "partial risk" does not exist). Finally, it allows for dealing with ambiguous situations for which partial ambiguity remains silent, such as E1 and E1000.

Following Jewitt and Mukerji (2017), the acts of betting on the color of the ball drawn from the ambiguous urns can be ordered in terms of their degree of ambiguity. Formally a more ambiguous relation is defined as follows

Definition (Jewitt and Mukerji, 2017) Let $\mathcal{P}$ be a class of preferences over a set A. Assume that a binary relation "more ambiguity averse" is given, which is a strict partial order. Assume that each $\succsim \in \mathcal{P}$ is related to an ambiguity neutral element of $\mathcal{P}$. Given two acts $f, g \in \mathrm{A}, f$ is a more ambiguous act than $g$ if the following conditions are satisfied:

(i) if $\succsim \in \mathcal{P}$ is ambiguity neutral, then $g \sim f$;

(ii) for all $\succsim_{A}, \succsim_{B} \in \mathcal{P}$ such that $\succsim_{A}$ is an ambiguity neutral preference and $\succsim_{B}$ is more (less) ambiguity averse than $\succsim_{A}$, we have $g \succsim_{B}\left(\precsim_{B}\right) f$.

According to this definition, an act $f$ is more ambiguous than an act $g$ if an ambiguityaverse DM prefers $g$ to $f$, but an ambiguity-neutral DM is indifferent between the acts. It should be clear, however, that this order of more ambiguous arises on the back of a specific relation on preferences. In the context of the smooth ambiguity model (i.e, when the preference class partially ordered by a more ambiguity-averse relation is characterized by a more concave function $\phi$ ), we can order the partial ambiguity situations presented in Table 2 exclusively in terms how much they are affected by ambiguity. In line with Observation 2, we obtain the following results

\section{Proposition 2.}

(i) Under $I_{n}$ and $T_{n}$, the higher the $n$, the more ambiguous is the situation,

(ii) Under $D_{n}$, the lower the $n$, the more ambiguous is the situation.

In addition, the ambiguous situations encountered in Thought Experiments 1 and 2-for which partial ambiguity remains silent-can now be classified using the more ambiguous relation. In particular, by letting $E_{N}$ denote the two-color ambiguous urn with a total number of balls $N$ in it, we have

Proposition 3. For ambiguous urns $E_{N}$, a larger $N$ is always preferred under ambiguity aversion.

Propositions 2 and 3 thus indicate whether the class of smooth ambiguity deems some acts to be more ambiguous than others. These results are achieved without further restriction on ambiguity and risk attitudes but with a fixed belief (within the families discussed 
previously). ${ }^{11}$ They make clear that the counterintuitive Corollary 1 , i.e. that full ambiguity may be more desirable than partial ambiguity, is due to the fact that, for example, the situation associated with the set $M=\left\{\frac{0}{100}, \frac{100}{100}\right\}$ is more ambiguous than the one associated with the set $M=\left\{\frac{0}{100}, \frac{1}{100}, \ldots, \frac{100}{100}\right\}$. Such a result is in line with Ellsberg's quote above: situations like the one with $M=\left\{\frac{0}{100}, \frac{100}{100}\right\}$ present a high degree of ambiguity, as the available information is highly conflicting and leads to expectations that differ widely.

Finally note that, in a recent study, Filiz-Ozbay et al. (2020) investigate experimentally whether the number of balls in Ellsberg's two-color urn matters for decisions. Specifically, they use binary comparisons between urns $E_{2}, E_{10}$, and $E_{1000}$ and show that the majority of subjects (around 60\%) prefer urns with larger $N$. In accordance with Proposition 3, they further show that this preference for the larger urn is mainly driven by ambiguity averse subjects.

\section{Concluding remarks}

The notion of partial ambiguity has been recently proposed to describe intermediate forms of ambiguous situations, lying between risk and full ambiguity. In this paper, I show that partial ambiguity, which implicitly relies on the quantity of information provided about the possible probability models describing the stochastic phenomenon of interest, may lead to counterintuitive results. Rather, I argue that the quality of the information is ultimately what determines whether an ambiguous situation is deemed more desirable than another. Following this idea, I propose a reinterpretation of partial ambiguity in the light of the more ambiguous relation proposed by Jewitt and Mukerji (2017). I show that, in the cases in which full ambiguity may surprisingly prove more desirable than partial ambiguity, it is just because it is less ambiguous.

\footnotetext{
${ }^{11}$ As noted by Jewitt and Mukerji (2017, p. 215), "Fixing belief is natural and necessary given that we are in a framework with subjective belief on states and choice objects are acts, rather than lotteries involving given distributions on outcomes. In this framework one needs such a restriction even when working with expected utility (say, to investigate risk orders on acts) since under different beliefs the same act will induce correspondingly different lotteries over outcomes; an act that is riskier under one belief can be the opposite under another belief."
} 


\section{Appendix}

\section{A Proofs}

Proof of Proposition 2 Directly follows from Observation 1 and the fact that all partial ambiguity situations are equivalent under ambiguity neutrality (i.e., under SEU).

Proof of Proposition 3 To prove Proposition 3, we use the graphical illustration presented in Figure A.1. It depicts the cumulative distribution function (CDF) over the set of possible probability models when $N=\{1,10,100,1000\}$. As the figure shows, the mean is the same for all distributions. The case $E_{1}$ corresponds to the situation in which the two extreme probability models $P(r)=0$ and $P(r)=1$ both receive a subjective weight 0.5 (symmetry condition). It can then be easily shown that its CDF is second-order stochastically dominated by any of the CDFs presented in the figure. Assuming ambiguity aversion, this makes $E_{1}$ the least preferred option among all the $E_{N}$. In the same vein, the CDF of $E_{1000}$ second-order stochastically dominates (SOSD) all the other CDFs, making the bet on the urn with 1000 balls the most preferred among the four under ambiguity aversion. Similarly, when considering the intermediate situation $E_{100}$, in which each of the 101 possible probability models $P(r)=\{0,0.01, \ldots, 1\}$ receive an equal weight $1 / 101$, represented by the step function in Figure A.1, we can show that the distribution of $E_{100}$ SOSD that of $E_{10}$, which itself SOSD that of $E_{1}$. In the limit, the uniform distribution over all the possible compositions (in the case of $E_{N}$ with $N \rightarrow \infty$, represented by the 45-degree line) SOSD the distribution of any of the $E_{N}$ with a finite $N$. 


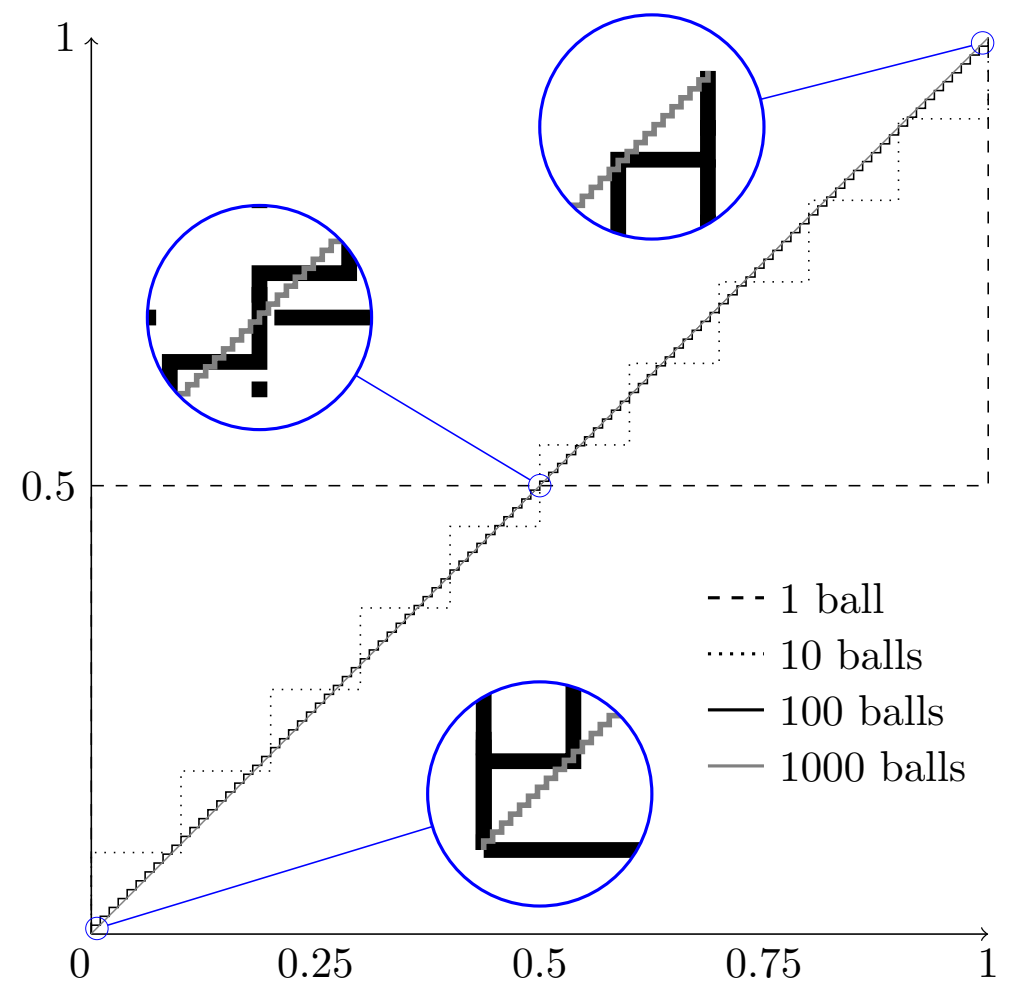

Figure A.1: Cumulative distribution functions of different bets over the set of possible models describing $E_{1}, E_{10}, E_{100}$, and $E_{1000}$ 


\section{References}

Arrow, K. J. (1951). Alternative approaches to the theory of choice in risk-taking situations. Econometrica 19, 404-437.

Aydogan, I., L. Berger, V. Bosetti, and N. Liu (2020). Three layers of uncertainty and the role of model misspecification. iRisk Working Paper 2020-01, IESEG School of M anagement.

Berger, L. and V. Bosetti (2020). Characterizing ambiguity attitudes using model uncertainty. Journal of Economic Behavior and Organization 180, 621-637.

Cerreia-Vioglio, S., F. Maccheroni, M. Marinacci, and L. Montrucchio (2013). Classical subjective expected utility. Proceedings of the National Academy of Sciences 110(17), $6754-6759$.

Chew, S. H., B. Miao, and S. Zhong (2017). Partial ambiguity. Econometrica 85(4), 1239-1260.

Einhorn, H. J. and R. M. Hogarth (1985). Ambiguity and uncertainty in probabilistic inference. Psychological review 92(4), 433.

Ellsberg, D. (1961). Risk, ambiguity, and the Savage axioms. The Quarterly Journal of Economics 75, 643-669.

Filiz-Ozbay, E., H. Gulen, Y. Masatlioglu, and E. Ozbay (2020). Comparing ambiguous urns with different sizes. Available at SSRN 3183973.

Hansen, L. P. (2014). Nobel lecture: Uncertainty outside and inside economic models. Journal of Political Economy 122(5), 945-987.

Hansen, L. P. and M. Marinacci (2016). Ambiguity aversion and model misspecification: An economic perspective. Statistical Science 31, 511-515.

Jewitt, I. and S. Mukerji (2017). Ordering ambiguous acts. Journal of Economic Theory 171, 213-267.

Keynes, J. M. (1921). A treatise on probability. Macmillan and Company, limited.

Klibanoff, P., M. Marinacci, and S. Mukerji (2005). A smooth model of decision making under ambiguity. Econometrica 73, 1849-1892.

Kops, C. and I. Pasichnichenko (2020). A test of information aversion.

Marinacci, M. (2015). Model uncertainty. Journal of the European Economic Association 13(6), 1022-1100.

Rothschild, M. and J. Stiglitz (1970). Increasing risk: I. a definition. Journal of Economic Theory 2(3), 225-243.

Savage, L. (1954). The Foundations of Statistics. New York: J. Wiley. second revised edition, 1972.

Schmeidler, D. (1989). Subjective probability and expected utility without additivity. Econometrica 57(3), 571-587.

Segal, U. (1987). The ellsberg paradox and risk aversion: An anticipated utility approach. International Economic Review, 175-202.

Seo, K. (2009). Ambiguity and second-order belief. Econometrica 77(5), 1575-1605.

Shishkin, D. and P. Ortoleva (2020). Ambiguous information and dilation: An experiment. Technical report, Tech. rep., Mimeo, Princeton University. 